\title{
Existence of Positive Solutions for Multipoint Boundary Value Problem with $p$-Laplacian on Time Scales
}

\author{
Meng Zhang, ${ }^{1}$ Shurong Sun, ${ }^{1}$ and Zhenlai Han ${ }^{1,2}$ \\ ${ }^{1}$ School of Science, University of Jinan, Jinan, Shandong 250022, China \\ ${ }^{2}$ School of Control Science and Engineering, Shandong University, Jinan, Shandong 250061, China
}

Correspondence should be addressed to Shurong Sun, sshrong@163.com

Received 11 March 2009; Accepted 8 May 2009

Recommended by Victoria Otero-Espinar

We consider the existence of positive solutions for a class of second-order multi-point boundary value problem with $p$-Laplacian on time scales. By using the well-known Krasnosel'ski's fixedpoint theorem, some new existence criteria for positive solutions of the boundary value problem are presented. As an application, an example is given to illustrate the main results.

Copyright (C) 2009 Meng Zhang et al. This is an open access article distributed under the Creative Commons Attribution License, which permits unrestricted use, distribution, and reproduction in any medium, provided the original work is properly cited.

\section{Introduction}

The theory of time scales has become a new important mathematical branch since it was introduced by Hilger [1]. Theoretically, the time scales approach not only unifies calculus of differential and difference equations, but also solves other problems that are a mix of stop start and continuous behavior. Practically, the time scales calculus has a tremendous potential for application, for example, Thomas believes that time scales calculus is the best way to understand Thomas models populations of mosquitoes that carry West Nile virus [2]. In addition, Spedding have used this theory to model how students suffering from the eating disorder bulimia are influenced by their college friends; with the theory on time scales, they can model how the number of sufferers changes during the continuous college term as well as during long breaks [2]. By using the theory on time scales we can also study insect population, biology, heat transfer, stock market, epidemic models [2-6], and so forth. At the same time, motivated by the wide application of boundary value problems in physical and applied mathematics, boundary value problems for dynamic equations with $p$-Laplacian on time scales have received lots of interest [7-16]. 
In [7], Anderson et al. considered the following three-point boundary value problem with $p$-Laplacian on time scales:

$$
\begin{gathered}
\left(\varphi_{p}\left(u^{\Delta}(t)\right)\right)^{\nabla}+c(t) f(u(t))=0, \quad t \in(a, b), \\
u(a)-B_{0}\left(u^{\Delta}(v)\right)=0, \quad u^{\Delta}(b)=0,
\end{gathered}
$$

where $v \in(a, b), f \in C_{\mathrm{ld}}([0, \infty),[0, \infty)), c \in C_{\mathrm{ld}}([a, b],[0, \infty))$, and $K_{m} x \leq B_{0}(x) \leq K_{M} x$ for some positive constants $K_{m}, K_{M}$. They established the existence results for at least one positive solution by using a fixed point theorem of cone expansion and compression of functional type.

For the same boundary value problem, He in [8] using a new fixed point theorem due to Avery and Henderson obtained the existence results for at least two positive solutions.

In [9], Sun and Li studied the following one-dimensional $p$-Laplacian boundary value problem on time scales:

$$
\begin{gathered}
\left(\varphi_{p}\left(u^{\Delta}(t)\right)\right)^{\Delta}+h(t) f\left(u^{\sigma}(t)\right)=0, \quad t \in[a, b], \\
u(a)-B_{0}\left(u^{\Delta}(a)\right)=0, \quad u^{\Delta}(\sigma(b))=0,
\end{gathered}
$$

where $h(t)$ is a nonnegative rd-continuous function defined in $[a, b]$ and satisfies that there exists $t_{0} \in[a, b]$ such that $h\left(t_{0}\right)>0, f(u)$ is a nonnegative continuous function defined on $[0, \infty), B_{1} x \leq B_{0}(x) \leq B_{2} x$ for some positive constants $B_{1}, B_{2}$. They established the existence results for at least single, twin, or triple positive solutions of the above problem by using Krasnosel'skii's fixed point theorem, new fixed point theorem due to Avery and Henderson and Leggett-Williams fixed point theorem.

For the Sturm-Liouville-like boundary value problem, in [17] Ji and Ge investigated a class of Sturm-Liouville-like four-point boundary value problem with $p$-Laplacian:

$$
\begin{aligned}
& \left(\varphi_{p}\left(u^{\prime}(t)\right)\right)^{\prime}+f(t, u(t))=0, \quad t \in(0,1), \\
& u(0)-\alpha u^{\prime}(\xi)=0, \quad u(1)+\beta u^{\prime}(\eta)=0,
\end{aligned}
$$

where $\xi<\eta, f \in C([0,1] \times[0, \infty),[0, \infty))$. By using fixed-point theorem for operators on a cone, they obtained some existence of at least three positive solutions for the above problem. However, to the best of our knowledge, there has not any results concerning the similar problems on time scales.

Motivated by the above works, in this paper we consider the following multi-point boundary value problem on time scales:

$$
\begin{gathered}
\left(\varphi_{p}\left(u^{\Delta}(t)\right)\right)^{\Delta}+h(t) f(u(t))=0, \quad t \in[a, b]_{\mathbb{T}}, \\
\alpha u(a)-\beta u^{\Delta}(\xi)=0, \quad \gamma u\left(\sigma^{2}(b)\right)+\delta u^{\Delta}(\eta)=0, \quad u^{\Delta}(\theta)=0,
\end{gathered}
$$


where $\mathbb{T}$ is a time scale, $\varphi_{p}(u)=|u|^{p-2} u, p>1, \alpha>0, \beta \geq 0, \gamma>0, \delta \geq 0, a<\xi<\theta<\eta<b$, and we denote $\left(\varphi_{p}\right)^{-1}=\varphi_{q}$ with $1 / p+1 / q=1$.

In the following, we denote $[a, b]:=[a, b]_{\mathbb{T}}=[a, b] \cap \mathbb{T}$ for convenience. And we list the following hypotheses:

$\left(C_{1}\right) f(u)$ is a nonnegative continuous function defined on $[0, \infty)$;

$\left(C_{2}\right) h:\left[a, \sigma^{2}(b)\right] \rightarrow[0, \infty)$ is rd-continuous with $h \cdot f \not \equiv 0$.

\section{Preliminaries}

In this section, we provide some background material to facilitate analysis of problem (1.4).

Let the Banach space $E=\left\{u:\left[a, \sigma^{2}(b)\right] \rightarrow \mathbb{R}\right.$ is rd-continuous $\}$ be endowed with the norm $\|u\|=\sup _{t \in\left[a, \sigma^{2}(b)\right]}|u(t)|$ and choose the cone $P \subset E$ defined by

$$
P=\left\{u \in E: u(t) \geq 0, t \in\left[a, \sigma^{2}(b)\right], u^{\Delta \Delta}(t) \leq 0, t \in[a, b]\right\} .
$$

It is easy to see that the solution of BVP (1.4) can be expressed as

$$
u(t)= \begin{cases}\frac{\beta}{\alpha} \varphi_{q}\left(\int_{\xi}^{\theta} h(r) f(u(r)) \Delta r\right)+\int_{a}^{t} \varphi_{q}\left(\int_{s}^{\theta} h(r) f(u(r)) \Delta r\right) \Delta s, & a \leq t \leq \theta, \\ \frac{\delta}{\gamma} \varphi_{q}\left(\int_{\theta}^{\eta} h(r) f(u(r)) \Delta r\right)+\int_{t}^{\sigma^{2}(b)} \varphi_{q}\left(\int_{\theta}^{s} h(r) f(u(r)) \Delta r\right) \Delta s, & \theta \leq t \leq \sigma^{2}(b) .\end{cases}
$$

If $V_{1}=V_{2}$, where

$$
\begin{aligned}
V_{1} & =\frac{\beta}{\alpha} \varphi_{q}\left(\int_{\xi}^{\theta} h(r) f(u(r)) \Delta r\right)+\int_{a}^{\theta} \varphi_{q}\left(\int_{s}^{\theta} h(r) f(u(r)) \Delta r\right) \Delta s, \\
V_{2} & =\frac{\delta}{\gamma} \varphi_{q}\left(\int_{\theta}^{\eta} h(r) f(u(r)) \Delta r\right)+\int_{\theta}^{\sigma^{2}(b)} \varphi_{q}\left(\int_{\theta}^{s} h(r) f(u(r)) \Delta r\right) \Delta s,
\end{aligned}
$$

we define the operator $A: P \rightarrow E$ by

$$
A u(t)= \begin{cases}\frac{\beta}{\alpha} \varphi_{q}\left(\int_{\xi}^{\theta} h(r) f(u(r)) \Delta r\right)+\int_{a}^{t} \varphi_{q}\left(\int_{s}^{\theta} h(r) f(u(r)) \Delta r\right) \Delta s, & a \leq t \leq \theta, \\ \frac{\delta}{\gamma} \varphi_{q}\left(\int_{\theta}^{\eta} h(r) f(u(r)) \Delta r\right)+\int_{t}^{\sigma^{2}(b)} \varphi_{q}\left(\int_{\theta}^{s} h(r) f(u(r)) \Delta r\right) \Delta s, & \theta \leq t \leq \sigma^{2}(b) .\end{cases}
$$


It is easy to see $\|u\|=u(\theta), A u(t) \geq 0$ for $t \in\left[a, \sigma^{2}(b)\right]$, and if $A u(t)=u(t)$, then $u(t)$ is the positive solution of BVP (1.4).

From the definition of $A$, for each $u \in P$, we have $A u \in P$, and $\|A u\|=A u(\theta)$.

In fact,

$$
(A u)^{\Delta}(t)= \begin{cases}\varphi_{q}\left(\int_{t}^{\theta} h(r) f(u(r)) \Delta r\right) \geq 0, & a \leq t \leq \theta \\ -\varphi_{q}\left(\int_{\theta}^{t} h(r) f(u(r)) \Delta r\right) \leq 0, & \theta \leq t \leq \sigma^{2}(b)\end{cases}
$$

is continuous and nonincreasing in $\left[a, \sigma^{2}(b)\right]$. Moreover, $\varphi_{q}(x)$ is a monotone increasing continuously differentiable function,

$$
\left(\int_{t}^{\theta} h(s) f(u(s)) \Delta s\right)^{\Delta}=\left(-\int_{\theta}^{t} h(s) f(u(s)) \Delta s\right)^{\Delta}=-h(t) f(u(t)) \leq 0,
$$

then by the chain rule on time scales, we obtain

$$
(A u)^{\Delta \Delta}(t) \leq 0
$$

so, $A: P \rightarrow P$.

For the notational convenience, we denote

$$
\begin{gathered}
L_{1}=\left(\frac{\beta}{\alpha}+\theta-a\right) \varphi_{q}\left(\int_{a}^{\theta} h(r) \Delta r\right), \\
L_{2}=\left(\frac{\delta}{r}+\sigma^{2}(b)-\theta\right) \varphi_{q}\left(\int_{\theta}^{\sigma^{2}(b)} h(r) \Delta r\right), \\
M_{1}=\frac{\beta}{\alpha} \varphi_{q}\left(\int_{\xi}^{\theta} h(r) \Delta r\right)+\int_{\xi}^{\theta} \varphi_{q}\left(\int_{s}^{\theta} h(r) \Delta r\right) \Delta s, \\
M_{2}=\frac{\delta}{\gamma} \varphi_{q}\left(\int_{\theta}^{\eta} h(r) \Delta r\right)+\int_{\theta}^{\eta} \varphi_{q}\left(\int_{\theta}^{s} h(r) \Delta r\right) \Delta s, \\
M_{3}=\min \left\{\frac{\xi-a}{\theta-a}, \frac{\sigma^{2}(b)-\eta}{\sigma^{2}(b)-\theta}\right\}, \\
M_{4}=\max \left\{\frac{\theta-a}{\xi-a}, \frac{\sigma^{2}(b)-\theta}{\sigma^{2}(b)-\eta}\right\} .
\end{gathered}
$$


Lemma 2.1. $A: P \rightarrow P$ is completely continuous.

Proof. First, we show that $A$ maps bounded set into bounded set.

Assume that $c>0$ is a constant and $u \in \overline{P_{c}}$. Note that the continuity of $f$ guarantees that there exists $K>0$ such that $f(u) \leq \varphi_{p}(K)$. So

$$
\begin{aligned}
\|A u\| & =A u(\theta) \\
& =\frac{\beta}{\alpha} \varphi_{q}\left(\int_{\xi}^{\theta} h(r) f(u(r)) \Delta r\right)+\int_{a}^{\theta} \varphi_{q}\left(\int_{s}^{\theta} h(r) f(u(r)) \Delta r\right) \Delta s \\
& \leq \frac{\beta}{\alpha} \varphi_{q}\left(\int_{a}^{\theta} h(r) \varphi_{p}(K) \Delta r\right)+\int_{a}^{\theta} \varphi_{q}\left(\int_{a}^{\theta} h(r) \varphi_{p}(K) \Delta r\right) \Delta s \\
& =K\left(\frac{\beta}{\alpha}+\theta-a\right) \varphi_{q}\left(\int_{a}^{\theta} h(r) \Delta r\right) \\
& =K L_{1}, \\
\|A u\| & =A u(\theta) \\
& =\frac{\delta}{r} \varphi_{q}\left(\int_{\theta}^{\eta} h(r) f(u(r)) \Delta r\right)+\int_{\theta}^{\sigma^{2}(b)} \varphi_{q}\left(\int_{\theta}^{s} h(r) f(u(r)) \Delta r\right) \Delta s \\
& \leq \frac{\delta}{\gamma} \varphi_{q}\left(\int_{\xi}^{\sigma^{2}(b)} h(r) \varphi_{p}(K) \Delta r\right)+\int_{\theta}^{\sigma^{2}(b)} \varphi_{q}\left(\int_{\theta}^{\sigma^{2}(b)} h(r) \varphi_{p}(K) \Delta r\right) \Delta s \\
& =K\left(\frac{\delta}{r}+\sigma^{2}(b)-\theta\right) \varphi_{q}\left(\int_{\theta}^{\sigma^{2}(b)} h(r) \Delta r\right)
\end{aligned}
$$

That is, $A \overline{P_{c}}$ is uniformly bounded. In addition, it is easy to see

$$
\left|A u\left(t_{1}\right)-A u\left(t_{2}\right)\right| \leq \begin{cases}C\left|t_{1}-t_{2}\right| \varphi_{q}\left(\int_{a}^{\theta} h(r) \Delta r\right), & t_{1}, t_{2} \in[a, \theta], \\ C\left|t_{1}-t_{2}\right| \varphi_{q}\left(\int_{a}^{\sigma^{2}(b)} h(r) \Delta r\right), & t_{1} \in[a, \theta], t_{2} \in\left[\theta, \sigma^{2}(b)\right] \\ C\left|t_{1}-t_{2}\right| \varphi_{q}\left(\int_{\theta}^{\sigma^{2}(b)} h(r) \Delta r\right), & \text { or } t_{1}, t_{2} \in[a, \theta], t_{1} \in\left[\theta, \sigma^{2}(b)\right],\end{cases}
$$



compact.

So, by applying Arzela-Ascoli Theorem on time scales, we obtain that $A \overline{P_{c}}$ is relatively

Second, we will show that $A: \overline{P_{c}} \rightarrow P$ is continuous. Suppose that $\left\{u_{n}\right\}_{n=1}^{\infty} \subset \overline{P_{c}}$ and $u_{n}(t)$ converges to $u_{0}(t)$ uniformly on $\left[a, \sigma^{2}(b)\right]$. Hence, $\left\{A u_{n}(t)\right\}_{n=1}^{\infty}$ is uniformly bounded and equicontinuous on $\left[a, \sigma^{2}(b)\right]$. The Arzela-Ascoli Theorem on time scales tells us that there exists uniformly convergent subsequence in $\left\{A u_{n}(t)\right\}_{n=1}^{\infty}$. Let $\left\{A u_{n_{l}}(t)\right\}_{l=1}^{\infty}$ be a subsequence which converges to $v(t)$ uniformly on $\left[a, \sigma^{2}(b)\right]$. In addition,

$$
0 \leq A u_{n}(t) \leq \min \left\{K L_{1}, K L_{2}\right\}
$$

Observe that

$$
A u_{n}(t)= \begin{cases}\frac{\beta}{\alpha} \varphi_{q}\left(\int_{\xi}^{\theta} h(r) f\left(u_{n}(r)\right) \Delta r\right)+\int_{a}^{t} \varphi_{q}\left(\int_{s}^{\theta} h(r) f\left(u_{n}(r)\right) \Delta r\right) \Delta s, & a \leq t \leq \theta, \\ \frac{\delta}{\gamma} \varphi_{q}\left(\int_{\theta}^{\eta} h(r) f\left(u_{n}(r)\right) \Delta r\right)+\int_{t}^{\sigma^{2}(b)} \varphi_{q}\left(\int_{\theta}^{s} h(r) f\left(u_{n}(r)\right) \Delta r\right) \Delta s, & \theta \leq t \leq \sigma^{2}(b) .\end{cases}
$$

Inserting $u_{n_{l}}$ into the above and then letting $l \rightarrow \infty$, we obtain

$$
v(t)= \begin{cases}\frac{\beta}{\alpha} \varphi_{q}\left(\int_{\xi}^{\theta} h(r) f\left(u_{0}(r)\right) \Delta r\right)+\int_{a}^{t} \varphi_{q}\left(\int_{s}^{\theta} h(r) f\left(u_{0}(r)\right) \Delta r\right) \Delta s, & a \leq t \leq \theta, \\ \frac{\delta}{\gamma} \varphi_{q}\left(\int_{\theta}^{\eta} h(r) f\left(u_{0}(r)\right) \Delta r\right)+\int_{t}^{\sigma^{2}(b)} \varphi_{q}\left(\int_{\theta}^{s} h(r) f\left(u_{0}(r)\right) \Delta r\right) \Delta s, & \theta \leq t \leq \sigma^{2}(b),\end{cases}
$$

here we have used the Lebesgues dominated convergence theorem on time scales. From the definition of $A$, we know that $v(t)=A u_{0}(t)$ on $\left[a, \sigma^{2}(b)\right]$. This shows that each subsequence of $\left\{A u_{n}(t)\right\}_{n=1}^{\infty}$ uniformly converges to $A u_{0}(t)$. Therefore, the sequence $\left\{A u_{n}(t)\right\}_{n=1}^{\infty}$ uniformly converges to $A u_{0}(t)$. This means that $A$ is continuous at $u_{0} \in \overline{P_{c}}$. So, $A$ is continuous on $\overline{P_{c}}$ since $u_{0}$ is arbitrary. Thus, $A$ is completely continuous.

The proof is complete.

Lemma 2.2. Let $u \in P$, then $u(t) \geq((t-a) /(\theta-a))\|u\|$ for $t \in[a, \theta]$, and $u(t) \geq\left(\left(\sigma^{2}(b)-\right.\right.$ $\left.t) /\left(\sigma^{2}(b)-\theta\right)\right)\|u\|$ for $t \in\left[\theta, \sigma^{2}(b)\right]$.

Proof. Since $u^{\Delta \Delta}(t) \leq 0$, it follows that $u^{\Delta}(t)$ is nonincreasing. Hence, for $a<t<\theta$,

$$
\begin{aligned}
& u(t)-u(a)=\int_{a}^{t} u^{\Delta}(s) \Delta s \geq(t-a) u^{\Delta}(t) \\
& u(\theta)-u(t)=\int_{t}^{\theta} u^{\Delta}(s) \Delta s \leq(\theta-t) u^{\Delta}(t)
\end{aligned}
$$


from which we have

$$
u(t) \geq \frac{u(a)(\theta-t)+(t-a) u(\theta)}{\theta-a} \geq \frac{t-a}{\theta-a} u(\theta)=\frac{t-a}{\theta-a}\|u\|
$$

For $\theta \leq t \leq \sigma^{2}(b)$,

$$
\begin{gathered}
u\left(\sigma^{2}(b)\right)-u(t)=\int_{t}^{\sigma^{2}(b)} u^{\Delta}(s) \Delta s \leq\left(\sigma^{2}(b)-t\right) u^{\Delta}(t), \\
u(t)-u(\theta)=\int_{\theta}^{t} u^{\Delta}(s) \Delta s \geq(t-\theta) u^{\Delta}(t),
\end{gathered}
$$

we know

$$
u(t) \geq \frac{\left(\sigma^{2}(b)-t\right) u(\theta)+(t-\theta) u\left(\sigma^{2}(b)\right)}{\sigma^{2}(b)-\theta} \geq \frac{\sigma^{2}(b)-t}{\sigma^{2}(b)-\theta} u(\theta)=\frac{\sigma^{2}(b)-t}{\sigma^{2}(b)-\theta}\|u\| .
$$

The proof is complete.

Lemma 2.3 ([18]). Let $P$ be a cone in a Banach space $E$. Assum that $\Omega_{1}, \Omega_{2}$ are open subsets of $E$ with $0 \in \Omega_{1}, \overline{\Omega_{1}} \subset \Omega_{2}$. If

$$
A: P \cap\left(\overline{\Omega_{2}} \backslash \Omega_{1}\right) \rightarrow P
$$

is a completely continuous operator such that either

(i) $\|A x\| \leq\|x\|, \forall x \in P \cap \partial \Omega_{1}$ and $\|A x\| \geq\|x\|, \forall x \in P \cap \partial \Omega_{2}$, or

(ii) $\|A x\| \geq\|x\|, \forall x \in P \cap \partial \Omega_{1}$ and $\|A x\| \leq\|x\|, \forall x \in P \cap \partial \Omega_{2}$.

Then $A$ has a fixed point in $P \cap\left(\overline{\Omega_{2}} \backslash \Omega_{1}\right)$.

\section{Main Results}

In this section, we present our main results with respect to BVP (1.4).

For the sake of convenience, we define $f_{0}=\lim _{u \rightarrow 0^{+}}\left(f(u) / \varphi_{p}(u)\right), f_{\infty}=$ $\lim _{u \rightarrow \infty}\left(f(u) / \varphi_{p}(u)\right), i_{0}=$ number of zeros in the set $\left\{f_{0}, f_{\infty}\right\}$, and $i_{\infty}=$ number of $\infty$ in the set $\left\{f_{0}, f_{\infty}\right\}$.

Clearly, $i_{0}, i_{\infty}=0,1$, or 2 and there are six possible cases:

(i) $i_{0}=0$ and $i_{\infty}=0$;

(ii) $i_{0}=0$ and $i_{\infty}=1$;

(iii) $i_{0}=0$ and $i_{\infty}=2$; 
(iv) $i_{0}=1$ and $i_{\infty}=0$;

(v) $i_{0}=1$ and $i_{\infty}=1$;

(vi) $i_{0}=2$ and $i_{\infty}=0$.

Theorem 3.1. BVP (1.4) has at least one positive solution in the case $i_{0}=1$ and $i_{\infty}=1$.

Proof. First, we consider the case $f_{0}=0$ and $f_{\infty}=\infty$. Since $f_{0}=0$, then there exists $H_{1}>0$ such that $f(u) \leq \varphi_{p}(\varepsilon) \varphi_{p}(u)=\varphi_{p}(\varepsilon u)$, for $0<u \leq H_{1}$, where $\varepsilon$ satisfies

$$
\max \left\{\varepsilon L_{1}, \varepsilon L_{2}\right\} \leq 1
$$

If $u \in P$, with $\|u\|=H_{1}$, then

$$
\begin{aligned}
\|A u\| & =A u(\theta) \\
& =\frac{\beta}{\alpha} \varphi_{q}\left(\int_{\xi}^{\theta} h(r) f(u(r)) \Delta r\right)+\int_{a}^{\theta} \varphi_{q}\left(\int_{s}^{\theta} h(r) f(u(r)) \Delta r\right) \Delta s \\
& \leq \frac{\beta}{\alpha} \varphi_{q}\left(\int_{a}^{\theta} h(r) f(u(r)) \Delta r\right)+\int_{a}^{\theta} \varphi_{q}\left(\int_{a}^{\theta} h(r) f(u(r)) \Delta r\right) \Delta s \\
& \leq \frac{\beta}{\alpha} \varphi_{q}\left(\int_{a}^{\theta} h(r) \varphi_{p}(\varepsilon\|u\|) \Delta r\right)+\int_{a}^{\theta} \varphi_{q}\left(\int_{a}^{\theta} h(r) \varphi_{p}(\varepsilon\|u\|) \Delta r\right) \Delta s \\
& =\|u\| \varepsilon L_{1} \\
& \leq\|u\|, \\
\|A u\| & =A u(\theta) \\
& =\frac{\delta}{r} \varphi_{q}\left(\int_{\theta}^{\eta} h(r) f(u(r)) \Delta r\right)+\int_{\theta}^{\sigma^{2}(b)} \varphi_{q}\left(\int_{\theta}^{s} h(r) f(u(r)) \Delta r\right) \Delta s \\
& \leq \frac{\delta}{r} \varphi_{q}\left(\int_{\theta}^{\sigma^{2}(b)} h(r) f(u(r)) \Delta r\right)+\int_{\theta}^{\sigma^{2}(b)} \varphi_{q}\left(\int_{\theta}^{\sigma^{2}(b)} h(r) f(u(r)) \Delta r\right) \Delta s \\
& \leq \frac{\delta}{r} \varphi_{q}\left(\int_{\theta}^{\sigma^{2}(b)} h(r) \varphi_{p}(\varepsilon\|u\|) \Delta r\right)+\int_{\theta}^{\sigma^{2}(b)} \varphi_{q}\left(\int_{\theta}^{\sigma^{2}(b)} h(r) \varphi_{p}(\varepsilon\|u\|) \Delta r\right) \Delta s \\
& =\|u\| \varepsilon L_{2} \\
& \leq\|u\| .
\end{aligned}
$$

It follows that if $\Omega_{H_{1}}=\left\{u \in E:\|u\|<H_{1}\right\}$, then $\|A u\| \leq\|u\|$ for $u \in P \cap \partial \Omega_{H_{1}}$. 
Since $f_{\infty}=\infty$, then there exists $H_{2}^{\prime}>0$ such that $f(u) \geq \varphi_{p}(k) \varphi_{p}(u)=\varphi_{p}(k u)$, for $u \geq H_{2}^{\prime}$, where $k>0$ is chosen such that

$$
\min \left\{k \frac{\xi-a}{\theta-a} M_{1}, k \frac{\sigma^{2}(b)-\eta}{\sigma^{2}(b)-\theta} M_{2}\right\} \geq 1
$$

Set $H_{2}=\max \left\{2 H_{1},((\theta-a) /(\xi-a)) H_{2}^{\prime},\left(\left(\sigma^{2}(b)-\theta\right) /\left(\sigma^{2}(b)-\eta\right)\right) H_{2}^{\prime}\right\}$, and $\Omega_{H_{2}}=\{u \in$ $\left.E:\|u\|<H_{2}\right\}$.

If $u \in P$ with $\|u\|=H_{2}$, then

$$
\begin{gathered}
\min _{t \in[\xi, \theta]} u(t)=u(\xi) \geq \frac{\xi-a}{\theta-a}\|u\| \geq H_{2}^{\prime} \\
\min _{t \in[\theta, \eta]} u(t)=u(\eta) \geq \frac{\sigma^{2}(b)-\eta}{\sigma^{2}(b)-\theta}\|u\| \geq H_{2}^{\prime}
\end{gathered}
$$

So that

$$
\begin{aligned}
\|A u\| & =A u(\theta) \\
& =\frac{\beta}{\alpha} \varphi_{q}\left(\int_{\xi}^{\theta} h(r) f(u(r)) \Delta r\right)+\int_{a}^{\theta} \varphi_{q}\left(\int_{s}^{\theta} h(r) f(u(r)) \Delta r\right) \Delta s \\
& \geq \frac{\beta}{\alpha} \varphi_{q}\left(\int_{\xi}^{\theta} h(r) \varphi_{p}(k u) \Delta r\right)+\int_{\xi}^{\theta} \varphi_{q}\left(\int_{s}^{\theta} h(r) \varphi_{p}(k u) \Delta r\right) \Delta s \\
& \geq \frac{\beta}{\alpha} \varphi_{q}\left(\int_{\xi}^{\theta} h(r) \varphi_{p}\left(k \frac{\xi-a}{\theta-a}\|u\|\right) \Delta r\right)+\int_{\xi}^{\theta} \varphi_{q}\left(\int_{s}^{\theta} h(r) \varphi_{p}\left(k \frac{\xi-a}{\theta-a}\|u\|\right) \Delta r\right) \Delta s \\
& =\|u\| k \frac{\xi-a}{\theta-a} M_{1} \\
& \geq\|u\|, \\
\|A u\| & =A u(\theta)=\frac{\delta}{r} \varphi_{q}\left(\int_{\theta}^{\eta} h(r) f(u(r)) \Delta r\right)+\int_{\theta}^{\sigma^{2}(b)} \varphi_{q}\left(\int_{\theta}^{s} h(r) f(u(r)) \Delta r\right) \Delta s \\
& \geq \frac{\delta}{\gamma} \varphi_{q}\left(\int_{\theta}^{\eta} h(r) \varphi_{p}\left(k \frac{\sigma^{2}(b)-\eta}{\sigma^{2}(b)-\theta}\|u\|\right) \Delta r\right)+\int_{\theta}^{\eta} \varphi_{q}\left(\int_{\theta}^{s} h(r) \varphi_{p}\left(k \frac{\sigma^{2}(b)-\eta}{\sigma^{2}(b)-\theta}\|u\|\right) \Delta r\right) \Delta s \\
& =\|u\| k \frac{\sigma^{2}(b)-\eta}{\sigma^{2}(b)-\theta} M_{2} \\
& \geq\|u\| .
\end{aligned}
$$


In other words, if $u \in P \cap \partial \Omega_{H_{2}}$, then $\|A u\| \geq\|u\|$. Thus by (i) of Lemma 2.3, it follows that $A$ has a fixed point in $P \cap\left(\overline{\Omega_{H_{2}}} \backslash \Omega_{H_{1}}\right)$ with $H_{1} \leq\|u\| \leq H_{2}$.

Now we consider the case $f_{0}=\infty$ and $f_{\infty}=0$. Since $f_{0}=\infty$, there exists $H_{3}>0$, such that $f(u) \geq \varphi_{p}(m) \varphi_{p}(u)=\varphi_{p}(m u)$ for $0<u \leq H_{3}$, where $m$ is such that

$$
\min \left\{m M_{1} \frac{\xi-a}{\theta-a}, m M_{2} \frac{\sigma^{2}(b)-\eta}{\sigma^{2}(b)-\theta}\right\} \geq 1
$$

If $u \in P$ with $\|u\|=H_{3}$, then we have

$$
\begin{aligned}
\|A u\| & =A u(\theta) \\
& =\frac{\beta}{\alpha} \varphi_{q}\left(\int_{\xi}^{\theta} h(r) f(u(r)) \Delta r\right)+\int_{a}^{\theta} \varphi_{q}\left(\int_{s}^{\theta} h(r) f(u(r)) \Delta r\right) \Delta s \\
& \geq \frac{\beta}{\alpha} \varphi_{q}\left(\int_{\xi}^{\theta} h(r) \varphi_{p}\left(m \frac{\xi-a}{\theta-a}\|u\|\right) \Delta r\right)+\int_{\xi}^{\theta} \varphi_{q}\left(\int_{s}^{\theta} h(r) \varphi_{p}\left(m \frac{\xi-a}{\theta-a}\|u\|\right) \Delta r\right) \Delta s \\
& =\|u\| m \frac{\xi-a}{\theta-a} M_{1} \\
& \geq\|u\|, \\
\|A u\| & =A u(\theta) \\
& =\frac{\delta}{r} \varphi_{q}\left(\int_{\theta}^{\eta} h(r) f(u(r)) \Delta r\right)+\int_{\theta}^{\sigma^{2}(b)} \varphi_{q}\left(\int_{\theta}^{s} h(r) f(u(r)) \Delta r\right) \Delta s \\
& \geq \frac{\delta}{\gamma} \varphi_{q}\left(\int_{\theta}^{\eta} h(r) \varphi_{p}\left(m \frac{\sigma^{2}(b)-\eta}{\sigma^{2}(b)-\theta}\|u\|\right) \Delta r\right)+\int_{\theta}^{\eta} \varphi_{q}\left(\int_{\theta}^{s} h(r) \varphi_{p}\left(m \frac{\sigma^{2}(b)-\eta}{\sigma^{2}(b)-\theta}\|u\|\right) \Delta r\right) \Delta s \\
& =\|u\| m \frac{\sigma^{2}(b)-\eta}{\sigma^{2}(b)-\theta} M_{2} \\
& \geq\|u\| .
\end{aligned}
$$

Thus, we let $\Omega_{H_{3}}=\left\{u \in E:\|u\|<H_{3}\right\}$, so that $\|A u\| \geq\|u\|$ for $u \in P \cap \partial \Omega_{H_{3}}$.

Next consider $f_{\infty}=0$. By definition, there exists $H_{4}^{\prime}>0$ such that $f(u) \leq \varphi_{p}(\varepsilon) \varphi_{p}(u)=$ $\varphi_{p}(\varepsilon \mathcal{u})$ for $u \geq H_{4}^{\prime}$, where $\varepsilon>0$ satisfies

$$
\max \left\{\varepsilon L_{1}, \varepsilon L_{2}\right\} \leq 1
$$


Advances in Difference Equations

Suppose $f$ is bounded, then $f(u) \leq \varphi_{p}(K)$ for all $u \in[0, \infty)$, pick

$$
H_{4}=\max \left\{2 H_{3}, K L_{1}, K L_{2}\right\}
$$

If $u \in P$ with $\|u\|=H_{4}$, then

$$
\begin{aligned}
\|A u\| & =A u(\theta) \\
& =\frac{\beta}{\alpha} \varphi_{q}\left(\int_{\xi}^{\theta} h(r) f(u(r)) \Delta r\right)+\int_{a}^{\theta} \varphi_{q}\left(\int_{s}^{\theta} h(r) f(u(r)) \Delta r\right) \Delta s \\
& \leq \frac{\beta}{\alpha} \varphi_{q}\left(\int_{a}^{\theta} h(r) \varphi_{p}(K) \Delta r\right)+\int_{a}^{\theta} \varphi_{q}\left(\int_{a}^{\theta} h(r) \varphi_{p}(K) \Delta r\right) \Delta s \\
& =K L_{1} \\
& \leq H_{4} \\
& =\|u\|, \\
\|A u\| & =A u(\theta) \\
& =\frac{\delta}{\gamma} \varphi_{q}\left(\int_{\theta}^{\eta} h(r) f(u(r)) \Delta r\right)+\int_{\theta}^{\sigma^{2}(b)} \varphi_{q}\left(\int_{\theta}^{s} h(r) f(u(r)) \Delta r\right) \Delta s \\
& \leq \frac{\delta}{r} \varphi_{q}\left(\int_{\theta}^{\sigma^{2}(b)} h(r) \varphi_{p}(K) \Delta r\right)+\int_{\theta}^{\sigma^{2}(b)} \varphi_{q}\left(\int_{\theta}^{\sigma^{2}(b)} h(r) \varphi_{p}(K) \Delta r\right) \Delta s \\
& =K L_{2} \\
& \leq H_{4} \\
& =\|u\| .
\end{aligned}
$$

Now suppose $f$ is unbounded. From condition $\left(C_{1}\right)$, it is easy to know that there exists $H_{4} \geq \max \left\{2 H_{3}, H_{4}\right\}$ such that $f(u) \leq f\left(H_{4}\right)$ for $0 \leq u \leq H_{4}$. If $u \in P$ with $\|u\|=H_{4}$, then by using (3.8) we have

$$
\begin{aligned}
\|A u\| & =A u(\theta) \\
& =\frac{\beta}{\alpha} \varphi_{q}\left(\int_{\xi}^{\theta} h(r) f(u(r)) \Delta r\right)+\int_{a}^{\theta} \varphi_{q}\left(\int_{s}^{\theta} h(r) f(u(r)) \Delta r\right) \Delta s \\
& \leq \frac{\beta}{\alpha} \varphi_{q}\left(\int_{a}^{\theta} h(r) f\left(H_{4}\right) \Delta r\right)+\int_{a}^{\theta} \varphi_{q}\left(\int_{a}^{\theta} h(r) f\left(H_{4}\right) \Delta r\right) \Delta s
\end{aligned}
$$




$$
\begin{aligned}
& \leq \frac{\beta}{\alpha} \varphi_{q}\left(\int_{a}^{\theta} h(r) \varphi_{p}\left(\varepsilon H_{4}\right) \Delta r\right)+\int_{a}^{\theta} \varphi_{q}\left(\int_{a}^{\theta} h(r) \varphi_{p}\left(\varepsilon H_{4}\right) \Delta r\right) \Delta s \\
& =H_{4} \varepsilon L_{1} \\
& \leq H_{4} \\
& =\|u\|, \\
\|A u\| & =A u(\theta) \\
& =\frac{\delta}{r} \varphi_{q}\left(\int_{\theta}^{\eta} h(r) f(u(r)) \Delta r\right)+\int_{\theta}^{\sigma^{2}(b)} \varphi_{q}\left(\int_{\theta}^{s} h(r) f(u(r)) \Delta r\right) \Delta s \\
& \leq \frac{\delta}{r} \varphi_{q}\left(\int_{\theta}^{\sigma^{2}(b)} h(r) f\left(H_{4}\right) \Delta r\right)+\int_{\theta}^{\sigma^{2}(b)} \varphi_{q}\left(\int_{\theta}^{\sigma^{2}(b)} h(r) f\left(H_{4}\right) \Delta r\right) \Delta s \\
& \leq \frac{\delta}{r} \varphi_{q}\left(\int_{\theta}^{\sigma^{2}(b)} h(r) \varphi_{p}\left(\varepsilon H_{4}\right) \Delta r\right)+\int_{\theta}^{\sigma^{2}(b)} \varphi_{q}\left(\int_{\theta}^{\sigma^{2}(b)} h(r) \varphi_{p}\left(\varepsilon H_{4}\right) \Delta r\right) \Delta s \\
& =H_{4} \varepsilon L_{2} \\
& \leq H_{4} \\
& =\|u\| .
\end{aligned}
$$

Consequently, in either case we take

$$
\Omega_{H_{4}}=\left\{u \in E:\|u\|<H_{4}\right\},
$$

so that for $u \in P \cap \partial \Omega_{H_{4}}$, we have $\|A u\| \geq\|u\|$. Thus by (ii) of Lemma 2.3, it follows that $A$ has a fixed point $u$ in $P \cap\left(\overline{\Omega_{H_{4}}} \backslash \Omega_{H_{3}}\right)$ with $H_{3} \leq\|u\| \leq H_{4}$.

The proof is complete.

Theorem 3.2. Suppose $i_{0}=0, i_{\infty}=1$, and the following conditions hold,

$\left(C_{3}\right)$ : there exists constant $p^{\prime}>0$ such that $f(u) \leq \varphi_{p}\left(p^{\prime} A_{1}\right)$ for $0 \leq u \leq p^{\prime}$, where

$$
A_{1}=\min \left\{L_{1}^{-1}, L_{2}^{-1}\right\}
$$


Advances in Difference Equations

$\left(C_{4}\right)$ : there exists constant $q^{\prime}>0$ such that $f(u) \geq \varphi_{p}\left(q^{\prime} A_{2}\right)$ for $u \in\left[M_{3} q^{\prime}, M_{3}\right]$, where

$$
A_{2}=\max \left\{M_{1}^{-1}, M_{2}^{-1}\right\}
$$

furthermore, $p^{\prime} \neq q^{\prime}$. Then BVP (1.4) has at least one positive solution $u$, such that $\|u\|$ lies between $p^{\prime}$ and $q^{\prime}$.

Proof. Without loss of generality, we may assume that $p^{\prime}<q^{\prime}$.

Let $\Omega_{p^{\prime}}=\left\{u \in E:\|u\|<p^{\prime}\right\}$, for any $u \in P \cap \partial \Omega_{p^{\prime}}$. In view of $\left(C_{3}\right)$ we have

$$
\begin{aligned}
\|A u\| & =A u(\theta) \\
& =\frac{\beta}{\alpha} \varphi_{q}\left(\int_{\xi}^{\theta} h(r) f(u(r)) \Delta r\right)+\int_{a}^{\theta} \varphi_{q}\left(\int_{s}^{\theta} h(r) f(u(r)) \Delta r\right) \Delta s \\
& \leq \frac{\beta}{\alpha} \varphi_{q}\left(\int_{a}^{\theta} h(r) \varphi_{p}\left(p^{\prime} A_{1}\right) \Delta r\right)+\int_{a}^{\theta} \varphi_{q}\left(\int_{a}^{\theta} h(r) \varphi_{p}\left(p^{\prime} A_{1}\right) \Delta r\right) \Delta s \\
& =p^{\prime} A_{1} L_{1} \\
& \leq p^{\prime} \\
\|A u\| & =A u(\theta) \\
& =\frac{\delta}{r} \varphi_{q}\left(\int_{\theta}^{\eta} h(r) f(u(r)) \Delta r\right)+\int_{\theta}^{\sigma^{2}(b)} \varphi_{q}\left(\int_{\theta}^{s} h(r) f(u(r)) \Delta r\right) \Delta s \\
& \leq \frac{\delta}{\gamma} \varphi_{q}\left(\int_{\theta}^{\sigma^{2}(b)} h(r) \varphi_{p}\left(p^{\prime} A_{1}\right) \Delta r\right)+\int_{\theta}^{\sigma^{2}(b)} \varphi_{q}\left(\int_{\theta}^{\sigma^{2}(b)} h(r) \varphi_{p}\left(p^{\prime} A_{1}\right) \Delta r\right) \Delta s \\
& =p^{\prime} A_{1} L_{2} \\
& \leq p^{\prime},
\end{aligned}
$$

which yields

$$
\|A u\| \leq\|u\| \quad \text { for } u \in P \cap \partial \Omega_{p^{\prime}} .
$$

Now set $\Omega_{q^{\prime}}=\left\{u \in E:\|u\|<q^{\prime}\right\}$ for $u \in P \cap \partial \Omega_{q^{\prime}}$, we have

$$
\begin{gathered}
\frac{\xi-a}{\theta-a} q^{\prime} \leq u(t) \leq q^{\prime} \quad \text { for } t \in[\xi, \theta], \\
\frac{\sigma^{2}(b)-\eta}{\sigma^{2}(b)-\theta} q^{\prime} \leq u(t) \leq q^{\prime} \quad \text { for } t \in[\theta, \eta] .
\end{gathered}
$$


Hence by condition $\left(C_{4}\right)$, we can get

$$
\begin{aligned}
\|A u\| & =A u(\theta) \\
& =\frac{\beta}{\alpha} \varphi_{q}\left(\int_{\xi}^{\theta} h(r) f(u(r)) \Delta r\right)+\int_{a}^{\theta} \varphi_{q}\left(\int_{s}^{\theta} h(r) f(u(r)) \Delta r\right) \Delta s \\
& \geq \frac{\beta}{\alpha} \varphi_{q}\left(\int_{\xi}^{\theta} h(r) \varphi_{p}\left(q^{\prime} A_{2}\right) \Delta r\right)+\int_{\xi}^{\theta} \varphi_{q}\left(\int_{s}^{\theta} h(r) \varphi_{p}\left(q^{\prime} A_{2}\right) \Delta r\right) \Delta s \\
& =q^{\prime} A_{2} M_{1} \\
& \geq q^{\prime}, \\
\|A u\| & =A u(\theta) \\
& =\frac{\delta}{r} \varphi_{q}\left(\int_{\theta}^{\eta} h(r) f(u(r)) \Delta r\right)+\int_{\theta}^{\sigma^{2}(b)} \varphi_{q}\left(\int_{\theta}^{s} h(r) f(u(r)) \Delta r\right) \Delta s \\
& \geq \frac{\delta}{r} \varphi_{q}\left(\int_{\theta}^{\eta} h(r) \varphi_{p}\left(q^{\prime} A_{2}\right) \Delta r\right)+\int_{\theta}^{\eta} \varphi_{q}\left(\int_{\theta}^{s} h(r) \varphi_{p}\left(q^{\prime} A_{2}\right)\right) \Delta s \\
& =q^{\prime} A_{2} M_{2} \\
& \geq q^{\prime} .
\end{aligned}
$$

So if we take $\Omega_{q^{\prime}}=\left\{u \in E:\|u\|<q^{\prime}\right\}$, then

$$
\|A u\| \geq\|u\|, \quad u \in P \cap \partial \Omega_{q^{\prime}} .
$$

Consequently, in view of $p^{\prime}<q^{\prime},(3.16)$, and (3.19), it follows from Lemma 2.3 that $A$ has a fixed point $u$ in $P \cap\left(\overline{\Omega_{q^{\prime}}} \backslash \Omega p^{\prime}\right)$. Moreover, it is a positive solution of (1.4) and $p^{\prime}<u<q^{\prime}$.

The proof is complete.

For the case $i_{0}=1, i_{\infty}=0$ or $i_{0}=0, i_{\infty}=1$ we have the following results.

Theorem 3.3. Suppose that $f_{0} \in\left[0, \varphi_{p}\left(A_{1}\right)\right)$ and $f_{\infty} \in\left(\varphi_{p}\left(M_{4} A_{2}\right), \infty\right)$ hold. Then BVP (1.4) has at least one positive solution.

Proof. It is easy to see that under the assumptions, the conditions $\left(C_{3}\right)$ and $\left(C_{4}\right)$ in Theorem 3.2 are satisfied. So the proof is easy and we omit it here.

Theorem 3.4. Suppose that $f_{0} \in\left(\varphi_{p}\left(M_{4} A_{2}\right), \infty\right)$ and $f_{\infty} \in\left[0, \varphi_{p}\left(A_{1}\right)\right)$ hold. Then BVP (1.4) has at least one positive solution.

Proof. Since $f_{0} \in\left(\varphi_{p}\left(M_{4} A_{2}\right), \infty\right)$, for $\varepsilon=f_{0}-\varphi_{p}\left(((\theta-a) /(\xi-a)) A_{2}\right)$, there exists a sufficiently small $q_{1}^{\prime}$ such that

$$
\frac{f(u)}{\varphi_{p}(u)} \geq f_{0}-\varepsilon=\varphi_{p}\left(\frac{\theta-a}{\xi-a} A_{2}\right), \quad u \in\left(0, q_{1}^{\prime}\right] .
$$


Thus, if $u \in\left(((\xi-a) /(\theta-a)) q_{1}^{\prime}, q_{1}^{\prime}\right]$, then we have

$$
f(u) \geq \varphi_{p}(u) \varphi_{p}\left(\frac{\theta-a}{\xi-a} A_{2}\right) \geq \varphi_{p}\left(q_{1}^{\prime} A_{2}\right)
$$

by the similar method, one can get if $u \in\left[\left(\left(\sigma^{2}(b)-\eta\right) /\left(\sigma^{2}(b)-\theta\right)\right) q_{2}^{\prime}, q_{2}^{\prime}\right]$, then

$$
f(u) \geq \varphi_{p}(u) \varphi_{p}\left(\frac{\sigma^{2}(b)-\theta}{\sigma^{2}(b)-\eta} A_{2}\right) \geq \varphi_{p}\left(q_{2}^{\prime} A_{2}\right)
$$

So, if we choose $q^{\prime}=\min \left\{q_{1}^{\prime}, q_{2}^{\prime}\right\}$, then for $u \in\left[M_{3} q^{\prime}, q^{\prime}\right]$, we have $f(u) \geq \varphi_{p}\left(q^{\prime} A_{2}\right)$, which yields condition $\left(C_{4}\right)$ in Theorem 3.2. such that

Next, by $f_{\infty} \in\left[0, \varphi_{p}\left(A_{1}\right)\right)$, for $\varepsilon=\varphi_{p}\left(A_{1}\right)-f_{\infty}$, there exists a sufficiently large $p^{\prime \prime}\left(>q^{\prime}\right)$

$$
\frac{f(u)}{\varphi_{p}(u)} \leq f_{\infty}+\varepsilon=\varphi_{p}\left(A_{1}\right), \quad u \in\left[p^{\prime \prime}, \infty\right)
$$

where we consider two cases.

Case 1. Suppose that $f$ is bounded, say

$$
f(u) \leq \varphi_{p}(K), \quad u \in[0, \infty) .
$$

In this case, take sufficiently large $p^{\prime}$ such that $p^{\prime} \geq \max \left\{K / A_{1}, p^{\prime \prime}\right\}$, then from (3.24), we know $f(u) \leq \varphi_{p}(K) \leq \varphi_{p}\left(A_{1} p^{\prime}\right)$ for $u \in\left(0, p^{\prime}\right]$, which yields condition $\left(C_{3}\right)$ in Theorem 3.2.

Case 2. Suppose that $f$ is unbounded. it is easy to know that there is $p^{\prime}>p^{\prime \prime}$ such that

$$
f(u) \leq f\left(p^{\prime}\right), \quad u \in\left[0, p^{\prime}\right]
$$

Since $p^{\prime}>p^{\prime \prime}$ then from (3.23) and (3.25), we get

$$
f(u) \leq f\left(p^{\prime}\right) \leq \varphi_{p}\left(p^{\prime} A_{1}\right), \quad u \in\left[0, p^{\prime}\right] .
$$

Thus, the condition $\left(C_{3}\right)$ of Theorem 3.2 is satisfied.

Hence, from Theorem 3.2, BVP (1.4) has at least one positive solution.

The proof is complete.

From Theorems 3.3 and 3.4, we have the following two results.

Corollary 3.5. Suppose that $f_{0}=0$ and the condition $\left(C_{4}\right)$ in Theorem 3.2 hold. Then BVP (1.4) has at least one positive solution. 
Corollary 3.6. Suppose that $f_{\infty}=0$ and the condition $\left(C_{4}\right)$ in Theorem 3.2 hold. Then BVP (1.4) has at least one positive solution.

Theorem 3.7. Suppose that $f_{0} \in\left(0, \varphi_{p}\left(A_{1}\right)\right)$ and $f_{\infty}=\infty$ hold. Then BVP (1.4) has at least one positive solution.

Proof. In view of $f_{\infty}=\infty$, similar to the first part of Theorem 3.1, we have

$$
\|A u\| \geq\|u\|, \quad u \in P \cap \partial \Omega_{H_{2}} .
$$

Since $f_{0} \in\left(0, \varphi_{p}\left(A_{1}\right)\right)$, for $\varepsilon=\varphi_{p}\left(A_{1}\right)-f_{0}>0$, there exists a sufficiently small $p^{\prime} \in\left(0, H_{2}\right)$ such that

$$
f(u) \leq\left(f_{0}+\varepsilon\right) \varphi_{p}(u)=\varphi_{p}\left(A_{1} u\right) \leq \varphi_{p}\left(A_{1} p^{\prime}\right), \quad u \in\left[0, p^{\prime}\right]
$$

Similar to the proof of Theorem 3.2, we obtain

$$
\|A u\| \leq\|u\|, \quad u \in P \cap \partial \Omega_{p^{\prime}} .
$$

The result is obtained, and the proof is complete.

Theorem 3.8. Suppose that $f_{\infty} \in\left(0, \varphi_{p}\left(A_{1}\right)\right)$ and $f_{0}=\infty$ hold. Then BVP (1.4) has at least one positive solution.

Proof. Since $f_{0}=\infty$, similar to the second part of Theorem 3.1, we have $\|A u\| \geq\|u\|$ for $u \in P \cap \partial \Omega_{H_{3}}$.

By $f_{\infty} \in\left(0, \varphi_{p}\left(A_{1}\right)\right)$, similar to the second part of proof of Theorem 3.4, we have $\|A u\| \leq\|u\|$ for $u \in P \cap \partial \Omega_{p^{\prime}}$, where $p^{\prime}>H_{3}$. Thus BVP (1.4) has at least one positive solution.

The proof is complete.

From Theorems 3.7 and 3.8, we can get the following corollaries.

Corollary 3.9. Suppose that $f_{\infty}=\infty$ and the condition $\left(C_{3}\right)$ in Theorem 3.2 hold. Then BVP (1.4) has at least one positive solution.

Corollary 3.10. Suppose that $f_{0}=\infty$ and the condition $\left(C_{3}\right)$ in Theorem 3.2 hold. Then BVP (1.4) has at least one positive solution.

Theorem 3.11. Suppose that $i_{0}=0, i_{\infty}=2$, and the condition $\left(C_{3}\right)$ of Theorem 3.2 hold. Then BVP (1.4) has at least two positive solutions $u_{1}, u_{2} \in P$ such that $0<\left\|u_{1}\right\|<p^{\prime}<\left\|u_{2}\right\|$.

Proof. By using the method of proving Theorems 3.1 and 3.2, we can deduce the conclusion easily, so we omit it here.

Theorem 3.12. Suppose that $i_{0}=2, i_{\infty}=0$, and the condition $\left(C_{4}\right)$ of Theorem 3.2 hold. Then BVP (1.4) has at least two positive solutions $u_{1}, u_{2} \in P$ such that $0<\left\|u_{1}\right\|<q^{\prime}<\left\|u_{2}\right\|$. 
Proof. Combining the proofs of Theorems 3.1 and 3.2, the conclusion is easy to see, and we omit it here.

\section{Applications and Examples}

In this section, we present a simple example to explain our result. When $\mathbb{T}=\mathbb{R}$,

$$
\begin{gathered}
u^{\prime}\left|u^{\prime}\right|=(1-t)(4-\arctan u), \quad 0<t<1, \\
u(0)=u^{\prime}\left(\frac{1}{4}\right), \quad u(1)=-u^{\prime}\left(\frac{1}{2}\right),
\end{gathered}
$$

where, $p=3, \alpha=\beta=\gamma=\delta=1, h(t)=1-t, f(u)=4-\arctan u$.

It is easy to see that the condition $\left(C_{1}\right)$ and $\left(C_{2}\right)$ are satisfied and

$$
f_{0}=\lim _{u \rightarrow 0^{+}} \frac{f(u)}{\varphi_{p}(u)}=\infty, \quad f_{\infty}=\lim _{u \rightarrow \infty} \frac{f(u)}{\varphi_{p}(u)}=0 .
$$

So, by Theorem 3.1, the BVP (4.1) has at least one positive solution.

\section{Acknowledgments}

This research is supported by the Natural Science Foundation of China (60774004), China Postdoctoral Science Foundation Funded Project (20080441126), Shandong Postdoctoral Funded Project (200802018), the Natural Science Foundation of Shandong (Y2007A27, Y2008A28), and the Fund of Doctoral Program Research of University of Jinan (B0621, XBS0843).

\section{References}

[1] S. Hilger, "Analysis on measure chains-a unified approach to continuous and discrete calculus," Results in Mathematics, vol. 18, no. 1-2, pp. 18-56, 1990.

[2] V. Spedding, "Taming nature's numbers," New Scientist, pp. 28-32, July 2003.

[3] M. A. Jones, B. Song, and D. M. Thomas, "Controlling wound healing through debridement," Mathematical and Computer Modelling, vol. 40, no. 9-10, pp. 1057-1064, 2004.

[4] D. M. Thomas, L. Vandemuelebroeke, and K. Yamaguchi, "A mathematical evolution model for phytoremediation of metals," Discrete and Continuous Dynamical Systems. Series B, vol. 5, no. 2, pp. 411-422, 2005.

[5] M. Bohner and A. Peterson, Dynamic Equations on Time Scales: An Introduction with Applications, Birkhäuser, Boston, Mass, USA, 2001.

[6] M. Bohner and A. Peterson, Eds., Advances in Dynamic Equations on Time Scales, Birkhäuser, Boston, Mass, USA, 2003.

[7] D. Anderson, R. Avery, and J. Henderson, "Existence of solutions for a one dimensional $p$-Laplacian on time-scales," Journal of Difference Equations and Applications, vol. 10, no. 10, pp. 889-896, 2004.

[8] Z. He, "Double positive solutions of three-point boundary value problems for $p$-Laplacian dynamic equations on time scales," Journal of Computational and Applied Mathematics, vol. 182, no. 2, pp. 304-315, 2005.

[9] H.-R. Sun and W.-T. Li, "Existence theory for positive solutions to one-dimensional $p$-Laplacian boundary value problems on time scales," Journal of Differential Equations, vol. 240, no. 2, pp. 217$248,2007$. 
[10] W.-T. Li and H. R. Sun, "Positive solutions for second-order $m$-point boundary value problems on time scales," Acta Mathematica Sinica, vol. 22, no. 6, pp. 1797-1804, 2006.

[11] H. R. Sun, Boundary value problems for dynamic equations on measure chains, Ph. D. thesis, Lanzhou University, Lanzhou, China, 2004.

[12] D. R. Anderson, "Existence of solutions for a first-order $p$-Laplacian BVP on time scales," Nonlinear Analysis: Theory, Methods \& Applications, vol. 69, no. 12, pp. 4521-4525, 2008.

[13] Y.-H. Su, W.-T. Li, and H.-R. Sun, "Positive solutions of singular $p$-Laplacian BVPs with sign changing nonlinearity on time scales," Mathematical and Computer Modelling, vol. 48, no. 5-6, pp. 845-858, 2008.

[14] X. Zhang and W. Ge, "Existence of positive solutions for a class of $m$-point boundary value problems," Advances in Difference Equations, vol. 2008, Article ID 845121, 9 pages, 2008.

[15] M. Feng, X. Li, and W. Ge, "Triple positive solutions of fourth-order four-point boundary value problems for $p$-Laplacian dynamic equations on time scales," Advances in Difference Equations, vol. 2008, Article ID 496078, 9 pages, 2008.

[16] C. Song, "Eigenvalue problems for $p$-Laplacian functional dynamic equations on time scales," Advances in Difference Equations, vol. 2008, Article ID 879140, 9 pages, 2008.

[17] D. Ji and W. Ge, "Existence of multiple positive solutions for Sturm-Liouville-like four-point boundary value problem with $p$-Laplacian," Nonlinear Analysis: Theory, Methods \& Applications, vol. 68, no. 9, pp. 2638-2646, 2008.

[18] D. J. Guo and V. Lakshmikantham, Nonlinear Problems in Abstract Cones, vol. 5 of Notes and Reports in Mathematics in Science and Engineering, Academic Press, San Diego, Calif, USA, 1988. 